\title{
Corneal endothelial changes under induced intraocular pressure elevation: a scanning and transmission electron microscopic study in rabbits
}

\author{
S. MELAMED, ${ }^{1}$ I. BEN-SIRA, ${ }^{1}$ AND Y. BEN-SHAUL ${ }^{2}$ \\ From the ${ }^{1}$ Department of Ophthalmology, Beilinson Medical Center, Tel-Aviv University, \\ Petah-Tikva, Israel, and the ${ }^{2}$ Department of Microbiology, the George S. Wise Faculty of \\ Life Sciences, Tel-Aviv University, Tel-Aviv, Israel
}

SUMMARY Intraocular pressure was artificially raised to $60-70 \mathrm{mmHg}$ in 7 albino rabbits for periods of 15 minutes to 4 hours. The corneal endothelium of these eyes was studied by transmission and scanning electron microscopy. A correletion between exposure time to elevated IOP, clinical signs observed by slit-lamp examination, and extent of morphological damage is clearly shown. In eyes exposed to high pressure for 15 and 30 minutes corneas remained transparent and only minimal changes could be detected by SEM, which consisted of small areas of cells with unevenness of their surface, occasional cellular ruptures, and diminution of cilia and microvilli. After 1-2 hours of exposure small, solitary corneal opacifications appeared. In these eyes more severe morphological changes affecting larger areas were observed, with additional cellular blebbing, excariocytosis, cellular rupture, disintegration, and disappearance seen in SEM. Thin sections revealed swelling of mitochondria, disorganisation of endoplasmic reticulum, and the existence of myelin bodies. In eyes exposed for 3 and 4 hours to high IOP diffuse corneal haziness, implying stromal oedema, appeared. In these eyes the areas affected were larger, the extent of damage being more severe. Many areas were bare of endothelium, surrounded by scattered cellular debris, and showed cells with ballooning surfaces and multiple ruptures. Even in severe cellular damage cellular junctions appeared intact. It is assumed that endothelial cells are more sensitive to IOP elevation than the cellular junctions and that injury to the active pump system due to morphological damage is responsible for the resultant corneal oedema.

It is widely accepted that endothelial integrity and metabolic activity are essential for continuous maintenance of corneal dehydration and transparency. On the one hand the endothelium is the site of a metabolically dependent fluid pump that counteracts fluid leakage, while on the other it acts as a barrier to solutes from the anterior chamber. ${ }^{1-5}$ Corneal oedema accompanying acute glaucoma was shown to result from noncompensated corneal endothelium. ${ }^{124-6}$ This noncompensation, which allows excessive flow of fluid to the corneal stroma, might be explained by two mechanisms: (1) High intraocular pressure (IOP) affects the metabolic active-pumping mechanism without any morphological changes, thus reducing resistance to aqueous flow to the stroma with resultant stromal oedema. ${ }^{5}$ (2) Elevated IOP causes morphological cellular

Correspondence to Dr S. Melamed. damage, thus reducing anatomically and functionally the barrier to flow of fluid into the stroma. In 47 eyes with chronic glaucoma decreased concentration of endothelial cells along with progressive atrophy and degeneration were described. ${ }^{6}$

More recently other investigators using scanning electron microscopy (SEM) and transmission electron microscopy (TEM) ${ }^{7-10}$ have shown various morphological changes which could be attributed to the nonfunctioning endothelium. Among these changes were flattening of cells, blebbing of cellular surface, intracellular vacuolisations, nuclear expulsion (excaryocytosis), and complete endothelial disorganisation or disappearance.

All these morphological changes were clearly shown by Svedbergh ${ }^{10}$ in monkey eyes exposed to moderate IOP (33-44 $\mathrm{mmHg}$ ) for 3-7 hours. However, in that study the IOP was elevated by adjusting the height of a reservoir containing mock 
aqueous humor connected to the anterior chamber. Although it is acceptable that high IOP was the main cause for the morphological changes described, some additional damage could be attributed to the perfusion of the anterior chamber. Perfusion of the cornea in vitro with Ringer bicarbonate without application of high pressure resulted in variable ultrastructural changes such as vacuolisation, mitochondrial and endoplasmic reticulum swelling, and complete intracellular disorganisation. ${ }^{11}$

The purpose of the present study was to investigate the extent and character of endothelial ultrastructural changes in relation to different exposure periods of elevated IOP and the extent of the resulting stromal oedema. In order to minimise possible damage due to perfusion and turbulence near the endothelium the IOP was artificially elevated by connecting a reservoir of normal saline to the vitreous through the pars plana.

\section{Materials and methods}

Seven albino rabbits of $2 \cdot 5-3.5 \mathrm{~kg}$ body weight were anaesthetised by injection of pentobarbitone sodium $(30 \mathrm{mg} / \mathrm{kg})$ intravenously. After deep anaesthesia was achieved the IOP was artificially elevated in the right eye, the left eye serving as a control. IOP elevation was carried out by connecting a reservoir of normal saline through the pars plana to the vitreous. The height of the reservoir was adjusted between 81 and $95 \mathrm{~cm}$, creating an IOP of $60-70 \mathrm{mmHg}$. The rate of IOP elevation was verified by measurement with hand-held applanation tonometer (Perkins).

To eliminate the possible effect of saline perfusion 2 control eyes were connected to the reservoir adjusted to a height of $10 \mathrm{~cm}$, keeping IOP within normal limits. During the period of IOP elevation we carried out slit-lamp examination, looking for signs of corneal oedema, ciliary congestion, flattening of the anterior chamber, pupillary diameter, and its reaction to light. Eyes examined were exposed to different period of IOP elevation, lasting 15 minutes, 30 minutes, 1 hour, 90 minutes, and 2,3 , and 4 hours.

At the end of the exposure time both eyes were enucleated and buttons of cornea were immediately cut from the globe. The corneas were immersed in $2 \%$ glutaraldehyde in $\mathbf{0 . 1} \mathrm{M}$ cacodylate buffer, $\mathrm{pH}$ $7 \cdot 4$, and fixed at $4^{\circ} \mathrm{C}$ overnight. For thin sectioning and TEM the tissue was postfixed in $2 \% \mathrm{OsO}_{4}$ in the same buffer and embedded in Epon. Thin sections on grids were stained with uranyl acetate and lead citrate. Preparations were examined and photographed in a Jeol-100B electron microscope at $80 \mathrm{kV}$. For SEM fixed pieces of cornea were

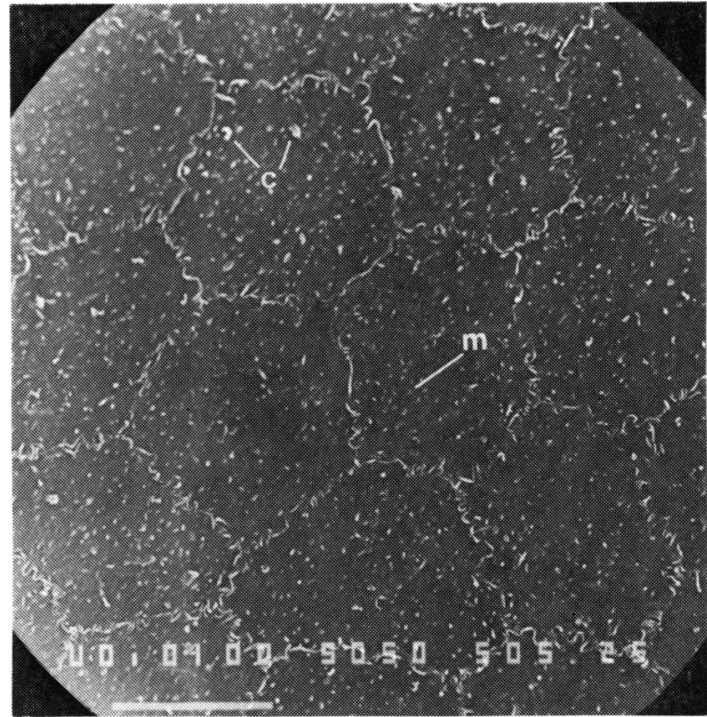

Fig. 1 Normal corneal endothelium (SEM). Cells are flat, hexagonal in shape, with randomly distributed microvilli (M) and cilia (C). Note the sharply demarcated intercellular borders. $\times 3000$.

postfixed with $\mathrm{OsO}_{4}$ as above, washed and dehydrated in a series of ethyl alcohols. Drying was carried out from liquid $\mathrm{CO}_{2}$ by the critical point method. After drying, preparation were coated with either gold or gold-paladium $(60-40 \%)$ by sputtering and photographed in a Jeol-S35 scanning electron microscope at $20-25 \mathrm{kV}$.

\section{Results}

On slit-lamp examination slight corneal opacification could be detected after 1-2 hours of exposure to high IOP. Diffuse stromal oedema appeared only after 3-4 hours of exposure. Ciliary congestion could be observed after 30 minutes, while pupillary mid-dilatation and nonreaction to light could be detected only after 1 hour. Flattening of the anterior chamber was noticed immediately.

In the control eyes (including the 2 eyes perfused with saline) the endothelial appearance resembled previously reported descriptions. ${ }^{12-15}$ In TEM the cells appeared flat, with numerous mitochondria and organised rough endoplasmic reticulum. Apical tight junctions were observed between cells with occasional pinocytotic vesicles and cilia on the cell surface. In SEM endothelial cells appeared flat, hexagonal in shape, with randomly distributed microvilli and cilia on the cell surface. Sharply demarcated, interdigitating cellular borders were also observed (Fig. 1). In eyes exposed for 15 and 


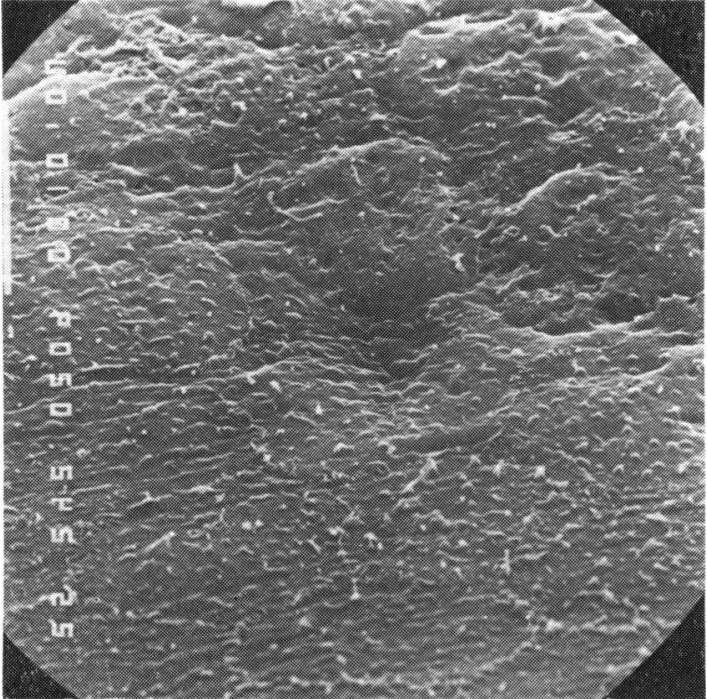

Fig. 2 Border area between nonaffected endothelium (bottom) and minimally damaged cells after 15 minutes of exposure to elevated IOP. Note unevenness of cellular surface and diminution of microvilli and cilia. $\times 3000$.

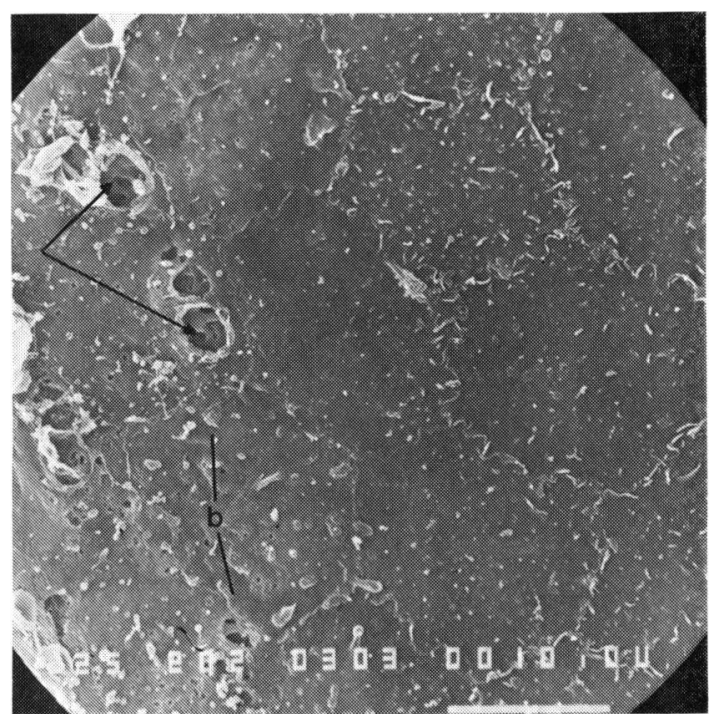

Fig. 3 Minimal damage to endothelium after 30 minutes of elevated IOP. Normally appeared endothelium (right) is adjacent to cells with attenuated cell surface,

blebbing (b), and cellular ruptures (arrows). $\times 3000$.

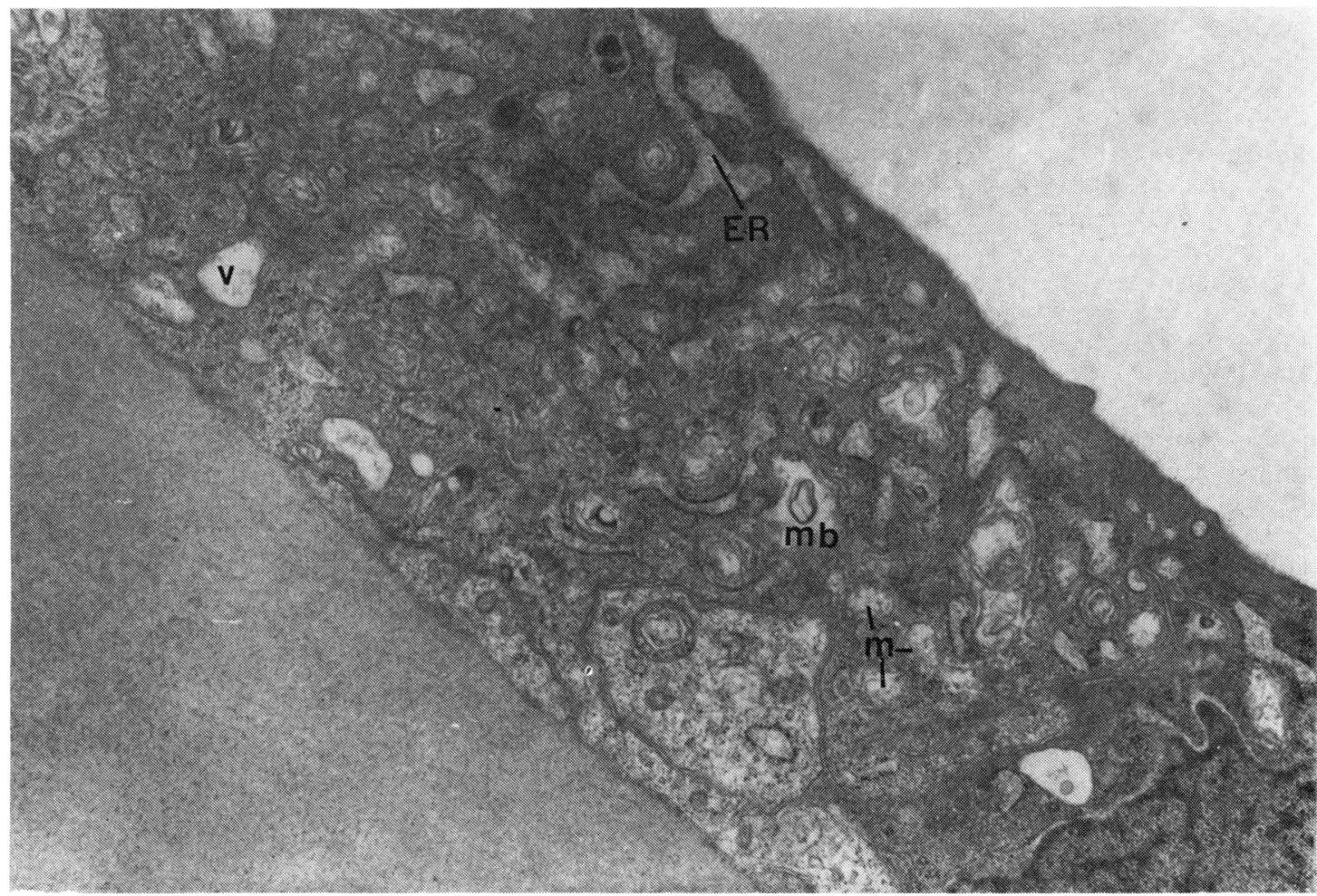

Fig. 4 Endothelial cell after exposure of 1 hour to high pressure. Distention and swelling of mitochondria (M) and endoplasmic reticulum (ER) is evident. Note vacuolisations $(V)$ and myelin body $(\mathrm{MB}) . \times 18000$. 


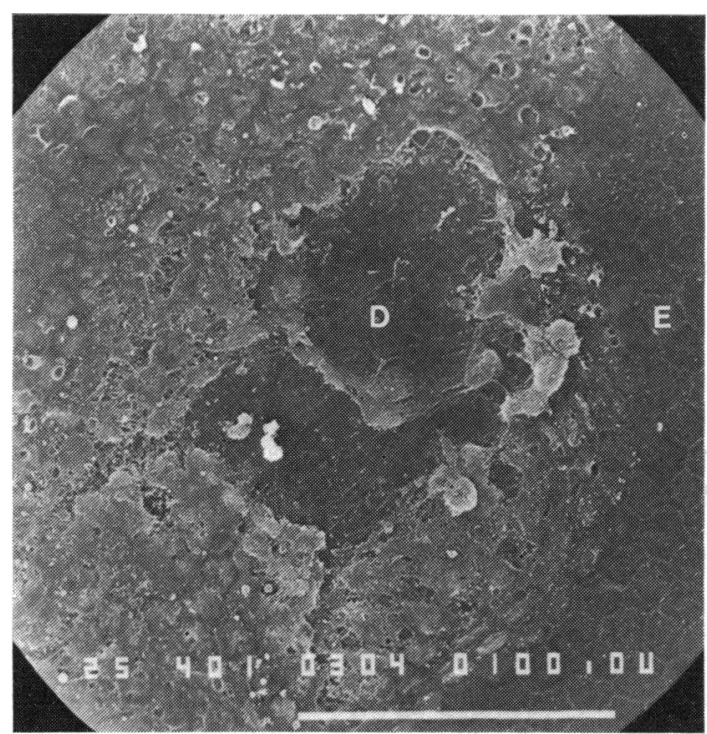

Fig. 5 An area of severely damaged endothelium after exposure of 4 hours to elevated IOP. The centre of insulated area is devoid of endothelium (D), surrounded by ruptures cells and cellular debris. At right normal endothelium (E) is seen and at left minimally damaged cells are shown. $\times 750$.

30 minutes to elevated IOP only anterior chamber flattening and ciliary congestion were observed by slit-lamp examination. No signs of corneal oedema or regional opacification were detected. Thin sections of the endothelial cells in these eyes were completely normal. In SEM only minimal changes could be seen. Most of the cells appeared normal, but in small, scattered areas unevenness of cellular surface along with slight swelling of cellular content were evident. Occasional ruptured cell surfaces were encountered. Cilia and microvilli were obseried less frequently (Figs. 2, 3).

In eyes exposed for 1-2 hours to high pressure, small solitary corneal opacifications could be seen on slit-lamp examination, along with pupillary mid-dilatation and nonreaction to light. Increased ciliary congestion and anterior chamber flattening were also observed. Eyes exposed to high IOP for 3 and 4 hours showed further deterioration of corneal opacification to more diffuse haziness, indicating progression of corneal oedema.

In thin sections affected cells showed disorganisation and swelling of endoplasmic reticulum and mitochondria along with vacuolisations and myelin bodies (probably evidence of cellular destruction) (Fig. 4). Despite intracellular damage junctional complexes appeared intact, unaffected by acutely elevated IOP.

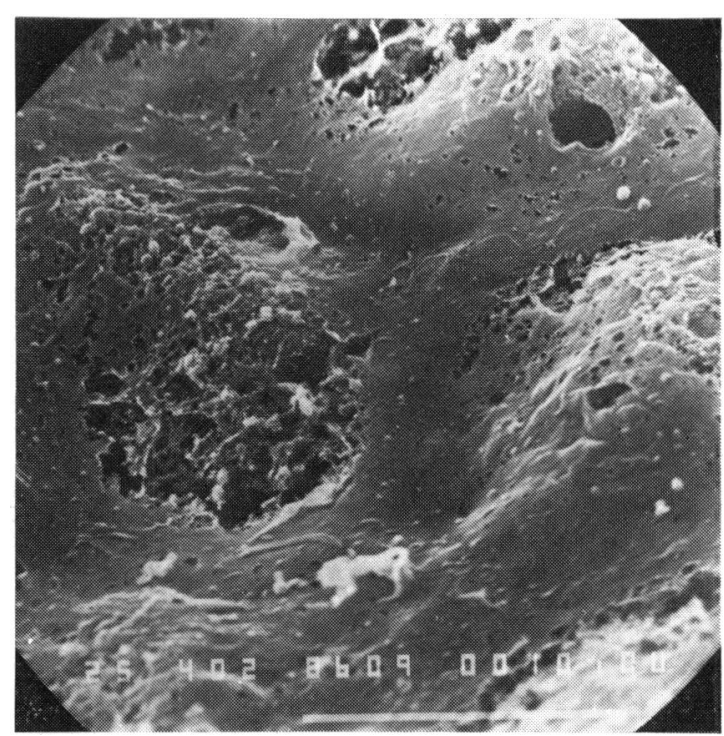

Fig. 6 Severely damaged endothelium. Ruptured cell containing empty crater after nuclear expulsion. $\times 6000$.

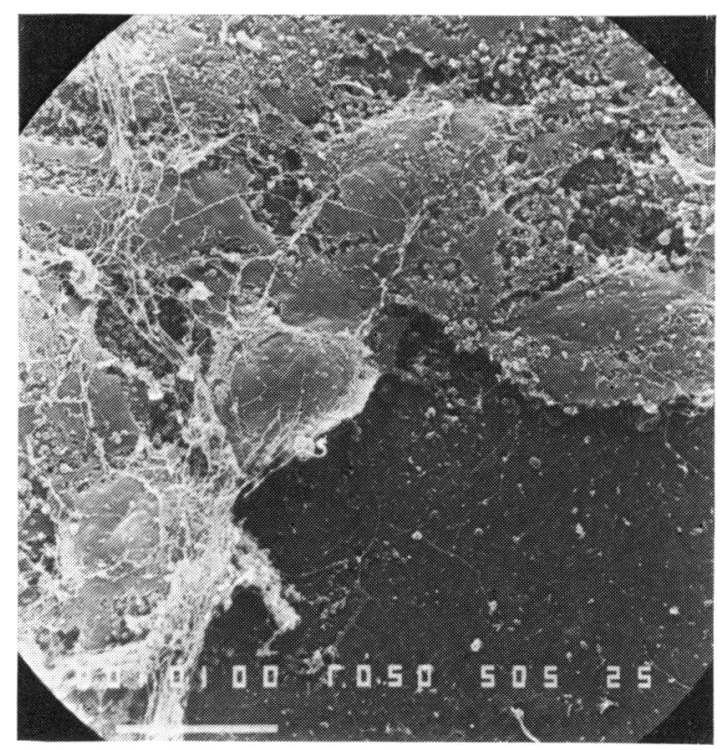

Fig. 7 Severely damaged endothelium. Bare area (Descemet's membrane) surrounded by ruptured disintegrated cells. $\times 3000$.

SEM, enabling the observation of large areas of the affected endothelium, disclosed different types of cellular damage. It was evident that larger areas of the endothelium were damaged when the eyes were exposed to elevated IOP for 3-4 h. Moreover, it was possible to monitor different types of mor. 


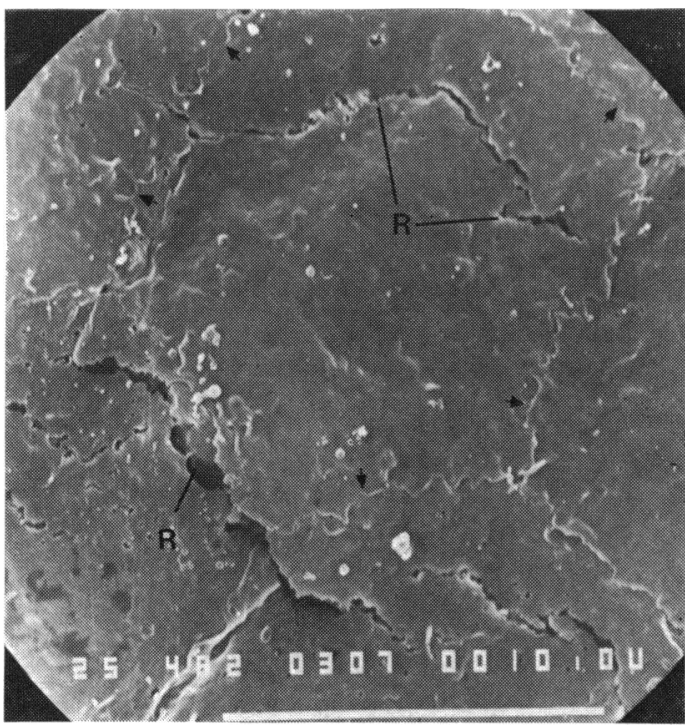

Fig. 8 Rupture of cellular surfaces. Rupture planes (R) leave intercellular junctions (arrow-heads) intact. $\times 7250$

phological changes in correlation with the exposure time to high pressure.

Along with the slight morphological changes (unevenness of cellular surface and diminution of cilia and microvilli) more severe changes appeared. These included blebbing and ballooning of cell surface, cellular swelling, nuclear expulsion (excaryocytosis), ruptured cells containing empty craters and scattered cellular content, disorganised cells and completely bare areas of Descemet's membrane devoid of endothelium, which had completely disappeared (Figs. 5-7). In some areas rupture of cellular surfaces could be noticed without blebbing or nuclear expulsion. It was evident in these cells that rupture planes passed through cellular surface only, leaving the intercellular borders intact (Fig. 8).

\section{Discussion}

In our study a good correlation between exposure time to elevated IOP, clinical signs, and extent of morphological changes was noted. After minimal exposure time (15 and 30 minutes) the cornea remained transparent, and only minimal morphological changes were detected in SEM (attenuation of cell surface, loss of cilia and microvilli, and occasional ruptures in small areas). After 1-2 hours small opacities appeared and more extensive damage was observed. In these eyes small areas of nuclear expulsion, cell rupture, and endothelial disintegration were encountered. In thin sections mitochondrial and endoplasmic reticulum swelling and disorganisation were evident, indicating damage to essential metabolic processes. In eyes exposed for 3 and 4 hours additional cellular disintegration, rupture and disappearance could be seen, along with progression of corneal opacification leading to diffuse oedema. It seems that cellular integration is essential to the proper action of the pump mechanism. The fact that despite severe cellular damage the tight junctions appeared intact stands out. It reconfirms previous postulations that these junctions are not the main contributors to the barrier against leakage of fluid. ${ }^{416}$

In the normal eyes the corneal endothelium acts as a barrier to fluid leakage into the stroma from the anterior chamber. This is carried out mainly by an active pumping system, which counteracts the passive leakage to the stroma. ${ }^{1-517}$ Ouabain, an inhibitor of $\mathrm{Na}^{+}-\mathrm{K}^{+}$activated ATPase system, had been demonstrated to cause corneal swelling when applied to the endothelium..$^{18}$ Further, an adequate ATP level was found to be required for transport activity. ${ }^{4}$ Thus it is believed that the active pump mechanism is $\mathrm{Na}^{+}-\mathrm{K}^{+}$ATPase dependent. In addition the existence of apical tight junctions between endothelial cells is believed to contribute to the 'sealing off' effect, though several investigators claim that a passive leakage through these junctions is possible and that this leakage is counteracted by the active pumping system. ${ }^{416}$ Perfusion of the cornea with $\mathrm{Ca}^{++}$-free medium caused corneal swelling and TEM disclosed disintegration of junctional complexes. ${ }^{1920}$ It was also found that large molecules (such as the colloidal tracer $\mathrm{ThO}_{2}$ ) can bypass the junctional complexes via pinocytotic vesicles. ${ }^{21}$

It is likely, however, that in addition to the main mechanism of active pumping the junctional complexes serve as passive barriers to fluid leakage, and pinocytosis helps in transferring large molecules across the endothelium, if necessary. Leber ${ }^{22}$ was the first to describe localised endothelial defects corresponding to spots of corneal opacity in a rabbit under IOP of $110 \mathrm{mmHg}$. Kaye et al., studying corneal behaviour in vitro under pressure of $60-80 \mathrm{mmHg}$, found the endothelial barrier to deteriorate before the metabolically dependent pump mechanism fails. TEM revealed no morphological changes (duration of high pressure was not stated). ${ }^{5}$ More recent morphological studies using TEM and SEM disclosed flattening of cells, blebbing of cellular surface, excaryocytosis, and endothelial disintegration. ${ }^{7-10}$

It seems that for a diffuse corneal oedema to develop a certain threshold of decompensation must be reached. We believe that unaffected endothelium in eyes exposed to high pressure for short 
periods can compensate for the rather small damaged and leaky areas, thus keeping corneal stroma dehydrated and transparent. However, if the extent of damage is more severe, as seen in eyes exposed for longer periods, the remaining functioning endothelium loses its ability to compensate for the larger leaking areas, and a diffuse corneal oedema results. In the rabbit cornea under IOP of 60-70 $\mathrm{mmHg}$ this decompensation appears after 3 to 4 hours of exposure.

This work was made possible by a grant from the Israeli Ministry of Health.

\section{References}

${ }^{1}$ Ytteborg J, Dohlman CH. Corneal edema and intraocular pressure. (1). Animal experiments. Arch Ophthalmol 1965; 74: 375-81.

${ }^{2}$ Ytteborg J, Dohlman CH. Corneal edema and intraocular pressure. (2). Clinical results. Arch Ophthalmol 1965; 74: 477-84.

${ }^{3}$ Donn A. Cornea and sclera. Arch Ophthalmol 1966; 75: 261-88.

${ }^{4}$ Fischbarg J. Active and passive properties of the corneal endothelium. Exp Eye Res 1973; 15: 615-38.

${ }^{5}$ Kaye GI, Sibley RC, Hoefle FB. Recent studies on the nature and function of the corneal endothelial barrier. Exp Eye Res 1973; 15: 585-613.

${ }^{6}$ Irvine AR. The role of the endothelium in bullous keratopathy. Arch Ophthalmol 1956; 56: 338-51.

${ }^{7}$ Hervouet F, George Y, Tusques J, Ertus M. Aspect de differentes structures oculaires humaines en microscope à balayage. Bull Soc Ophtalmol Fr 1972; 84: 603-20.

${ }^{8}$ Hervouet F, Ertus M. Scanning Electron Microscopic Studies of the Eye Structures. Paris: Masson, 1973; 12-13.

${ }^{9}$ Renard G, Galle P. Etude en microccopie à balayage de l'endothelium cornéen humain. Ann Oculist 1973; 206: 835-49.
${ }^{10}$ Svedbergh B. Effects of artificial intraocular pressure elevation on the corneal endothelium in the vervet monkey. Acta Ophthalmol 1975; 53: 839-55.

${ }^{11}$ Mccarey BE, Edelhauser HF, Van-Horn DL. Functional and structural changes in the corneal endothelium during in-vitro perfusion. Invest Ophthalmol 1973; 12: 410-17.

${ }^{12}$ Blumcke S, Morgenroth $\mathrm{K}$. The stereo ultrastructure of the external and internal surface of the cornea. J Ultrastruct Res 1967; 18: 502-18.

${ }^{13}$ Hogan $\mathrm{H}$, Alvarado J, Weddel J. Histology of the Human Eye. Philadelphia: Saunders, 1971; 102-11 and 171-8.

${ }^{14}$ Svedbergh B, Bill A. Scanning electron microscopic studies of the corneal endothelium in man and monkeys. Acta Ophthalmol 1972; 50: 321-36.

${ }^{15}$ Doughman DJ, Van-Horn D, Harris JE, Miller GE, Lindstrom R, Good RA. The ultrastructure of human organ cultured cornea. Arch Ophthalmol 1974; 92: 516-23.

${ }^{16}$ Mishima S, Kaye GI, Takahashi GH, Kudo T, Trenberth SM. In: Langham M, ed. The Cornea. Macromolecular Organization of a Connective Tissue. Baltimore: Johns Hopkins Press, 1969; 207.

${ }^{17}$ Maurice DM. The cornea and sclera. In: Davson $\mathrm{H}$, ed. The Eye. London and New York: Academic Press, 1969; 1: 489-600,

${ }^{18}$ Trenberth SM, Mishima S. The effect of ouabain on the rabbit corneal endothelium. Invest Ophthalmol 1968; 7: 44-52.

${ }^{19}$ Kaye GI, Mishima S, Cole DJ, Kaye NW. Studies on the cornea: VII. Effects of perfusion with a $\mathrm{Ca}^{++}$-free medium on the corneal endothelium. Invest Ophthalmol 1968; 7: 53-66.

${ }^{20}$ Kaye GI, Hoefle FB, Donn A. Studies on the cornea: VIII. Reversibility of the effects of in-vitro perfusion of the rabbit corneal endothelium with calcium-free medium. Invest Ophthalmol 1973; 12: 98-113.

${ }^{21}$ Kaye GI, Pappas GP, Donn A, Mallet N. Studies on the cornea: II. The uptake and transport of colloidal particles by the living rabbit cornea in-vitro. J Cell Biol 1962; 12: 481-501.

${ }^{22}$ Leber T. Studies uber den flussigkeitswechsel. Albrecht von Graeffes Arch Klin Ophthalmol 1873; 19: 87-185. 